\title{
LIE GROUPS SIMPLY ISOMORPHIC WITH NO LINEAR GROUP†
}

\section{BY GARRETT BIRKHOFF}

1. Introduction. One of the most interesting conjectures concerning finite continuous groups is the conjecture that every Lie group is topologically isomorphic with a group of matrices. The proof of this conjecture, even in the small, would establish the truth of the famous conjecture that every Lie group nucleus (or germ) is a piece of a Lie group. $\neq$ This makes it of interest to know that there exist Lie groups in the large, simply isomorphic even in the purely algebraic sense - and a fortiori topologically isomorphic in the large-with no group of matrices. It is to the proof of this fact that the present note is devoted.

2. The Basic Lemma. The proof ultimately rests on the following lemma.

LEMma 1. Let $\Gamma$ be any group of linear transformations. Suppose $\Gamma$ contains elements $S$ and $T$ whose commutator $R=S^{-1} T^{-1} S T$ is of prime order $p$, and satisfies $S R=R S, T R=R T$. Then $\Gamma$ is of degree at least $p$.

By the degree of $\Gamma$ is meant the dimensions - that is, the maximum number of linearly independent elements-of the linear space $\Sigma$ on which $\Gamma$ operates. The vector elements of $\Sigma$ will be written $x, y, z, \cdots$.

Proof. Since $R$ is of order $p, \Sigma$ contains a vector $x$ such that $R x=\alpha x$, where $\alpha$ is a primitive $p$ th root of unity. $\$$ Now let $\Lambda$ denote the linear subspace of all vectors $x \epsilon \Sigma$ satisfying $R x=\alpha x$. If $x \in \Lambda$, then

$$
R(S x)=S(R x)=S(\alpha x)=\alpha(S x) .
$$

$\dagger$ Presented to the Society, September 3, 1936.

$\$$ For a fuller description of the status of these conjectures, see [3], especially $\$ 17$. See also [1], p. 24, middle.

Numbers in square brackets refer to the Bibliography at the end of this paper.

$\S$ This follows from [4], Theorem 125 . 
That is, $S x \epsilon \Lambda$, showing that $S$, and likewise $T$, transforms $\Lambda$ into itself.

But now observe that within $\Lambda, S^{-1} T^{-1} S T=\alpha I$ (where $I$ denotes the identical matrix), whence $T^{-1} S T=S$. But $T^{-1} S T$ and $S$ have (see [4], Theorem 126) the same characteristic roots; hence so do $S$ and $\alpha S$, as linear transformations of $\Lambda$. Moreover since $S$ is non-singular, its characteristic roots are not zero; hence it has at least $p$ characteristic roots

$$
\mu_{0}, \alpha \mu_{0}, \cdots, \alpha^{p-1} \mu_{0}, \quad\left(\mu_{0} \neq 0\right) .
$$

It follows that $\Lambda$, and therefore $\Sigma$, has at least $p$ dimensions.

3. The Main Theorem. With the help of this lemma, one can easily exhibit a Lie group $\dagger$ simply isomorphic with no linear group. Let $G_{3}$ denote the group of all matrices

$$
M(x, y, z)=\left(\begin{array}{lll}
1 & x & z \\
0 & 1 & y \\
0 & 0 & 1
\end{array}\right) .
$$

And let $N$ denote the discrete normal subgroup of matrices $M(0,0, n), n$ any integer. Finally, let $G_{3}{ }^{*}=G_{3} / N$.

THEOREM $1 . G_{3}{ }^{*}$ is a Lie group simply isomorphic with no linear group.

Proof. That $G_{3}{ }^{*}$ is a Lie group can be verified directly, and also follows from general principles (see [1], p. 12). It remains to show that it is simply isomorphic with no linear group. But let $p$ be any prime; then the images $S$ of $M(1,0,0)$ and $T$ of $M(0,1 / p, 0)$ both commute with $R=S^{-1} T^{-1} S T$, which is the image of $M(0,0,1 / p)$ in $G_{3}$, while $R$ is of order $p$. Hence by Lemma 1 any linear group simply isomorphic with $G_{3}{ }^{*}$ would have to be of degree at least $p$, and since $p$ can be any prime, this is absurd.

Corollary. No group having $G_{3}^{*}$ as a subgroup is simply isomorphic with a linear group.

REMARK 1 . Since $G_{3}$ was a linear group, we see that there can

$\dagger$ By a Lie group we shall mean a connected finite continuous group in the usual sense; mixed groups will be excluded. See [1], p. 7 . 
exist no construction yielding, from representations of a given group as a linear group, non-trivial representations of its homomorphic images as linear groups.

Remark 2. As all the two-parameter Lie groups are known ([1], p. 25), and can be realized in the large as linear groups, $G_{3}^{*}$ is the simplest example of a group isomorphic in the large with no linear group.

4. Additional Definitions. In $\$ \S 4-6$ we shall show how to find whole families of Lie groups simply isomorphic with no linear group. But in order to do this, we shall need to recall some familiar parts of group theory.

By the central of a Lie group $G$ is meant the set of all elements of $G$ commuting with all other elements; this is a topologically closed and invariant subgroup of $G$.

Every Lie group $G$ has a Lie algebra $A$, defined from the set of the infinitesimal generators $U, V, \cdots$ of $G$ by regarding commutation (the taking of Poisson brackets) as a non-associative multiplication. Two Lie groups are locally isomorphic if and only if they have isomorphic Lie algebras ([2], [32.2]).

We shall call $G$ hypercentral if and only if the associated Lie algebra is nilpotent, in the sense that all products of sufficient length $r$ reduce to zero. This terminology is justified since $G$ is hypercentral if and only if, denoting by $Z_{1}$ the subgroup of $G$ consisting of the identity 0 , and by $Z_{k+1} / Z_{k}$ inductively the central of $G / Z_{k}$, we have $Z_{r}=G$. (Actually, $Z_{k}$ contains the invariant sub-algebra $A_{r-k}$ of $A$ consisting of linear combinations of products of lengths $\geqq(r-k)$.)

Remark. Any Lie group of triangular matrices $A=\left\|a_{i j}\right\|$ satisfying $a_{i i}=1$ and $a_{i j}=0$ if $i>j$ is hypercentral. All Poisson brackets of length exceeding its degree vanish.

5. A Theorem of Linear Groups. We shall now prove a result of some intrinsic interest, $\dagger$ which will be used in the sequel.

THEOREM 2. Let $U, V, W$ be any three matrices which satisfy $U V-V U=W, U W=W U, V W=W V$. Then the matrices $M\left(\lambda, \lambda^{\prime}, \lambda^{\prime \prime}\right)=\exp \left(\lambda U+\lambda^{\prime} V+\lambda^{\prime \prime} W\right)$ form a group $H$, topologically isomorphic in the large with $G_{3}$.

$\dagger$ Theorem 2 is especially interesting as $U, V, W$ have the formal properties of the position, momentum, and scalar operators $p, q, q p-p q$ of quantum mechanics. 
Explanation. As is customary, $\uparrow$ we use the notation

$$
\exp X=I+X+\frac{1}{2 !} X^{2}+\frac{1}{3 !} X^{3}+\cdots \text {. }
$$

Proof. Since for any $X,[\exp X] \circ[\exp (-X)]=I$, and since in $H$ one can prove the special identity

$$
\begin{aligned}
M\left(\lambda, \lambda^{\prime}, \lambda^{\prime \prime}\right) \circ M\left(\mu, \mu^{\prime}, \mu^{\prime \prime}\right) & \\
& =M\left(\lambda+\mu, \lambda^{\prime}+\mu^{\prime}, \lambda^{\prime \prime}+\mu^{\prime \prime}-\lambda^{\prime} \mu\right),
\end{aligned}
$$

clearly $H$ is a group. Further, (1) exhibits a continuous homomorphism $\ddagger$ of $G_{3}$ into $H$, since (1) expresses the rule for matrix multiplication in $G_{3}$.

We shall show that this is a topological isomorphism, in other words, that if $M\left(\lambda_{n}, \lambda_{n}^{\prime}, \lambda_{n}^{\prime \prime}\right) \equiv M_{n} \rightarrow M\left(\lambda, \lambda^{\prime}, \lambda^{\prime \prime}\right)$, then $\lambda_{n} \rightarrow \lambda, \lambda_{n}^{\prime} \rightarrow \lambda^{\prime}$, and $\lambda_{n}^{\prime \prime} \rightarrow \lambda^{\prime \prime}$. By group-homogeneity, it is sufficient to prove this for the special case $\lambda=\lambda^{\prime}=\lambda^{\prime \prime}=0$, $M\left(\lambda, \lambda^{\prime}, \lambda^{\prime \prime}\right)=I$. This is what we shall do.

The kernel of the demonstration is a well known theorem of Lie ([2], p. 163) which shows that one can so transform the matrices of $H$ that the generating matrices $U=\left\|u_{i j}\right\|$ and $V=\left\|v_{i j}\right\|$ are triangular, that is, satisfy $u_{i j}=v_{i j}=0$, if $i>j$. But now computing the matrix $W=U V-V U=\left\|w_{i j}\right\|$, we see that $w_{i j}=0$ unless $(j-i) \geqq 1$, while it is easily verified to be a general property of matrix multiplication, that if $Y$ and $Z$ are any two matrices such that $y_{i j}=0$ unless $(j-i) \geqq \alpha_{1}$, and $z_{i j}=0$ unless $(j-i) \geqq \alpha_{2}$, and $X=Y Z$, then $x_{i j}=0$ unless $(j-i) \geqq \alpha_{1}+\alpha_{2}$. It is a corollary that the non-zero coefficients of $W$ nearest the principal diagonal, that is, minimizing $(j-i)$ subject to $w_{i j} \neq 0$, appear as $\lambda^{\prime \prime} w_{i j}$ in exp $\left(\lambda^{\prime \prime} W\right)$, and so the subgroup of the $\exp \left(\lambda^{\prime \prime} W\right)$ is topologically isomorphic in the large with the translation-group $x \rightarrow x+t$.

Unless $\lambda_{n} \rightarrow 0$, we can, taking a suitable subsequence, assume without loss of generality that $1 / \lambda_{n}=\bar{\lambda}_{n} \rightarrow \bar{\lambda}$. But irrespective of $n$,

$$
M_{n}^{-1} \circ \exp \left(-\bar{\lambda}_{n} V\right) \circ M_{n} \circ \exp \left(\bar{\lambda}_{n} V\right)=\exp W \neq I ;
$$

† See J. von Neumann, Mathematische Zeitschrift, vol. 30 (1929), pp. 3-42.

$\ddagger$ For the distinction between continuous homomorphisms and topological isomorphisms see H. Freudenthal, Einige Sätze über topologische Gruppen, Annals of Mathematics, vol. 37 (1936), p. 46. 
while on the other hand, $M_{n} \rightarrow I$, exp $\left(\bar{\lambda}_{n} V\right) \rightarrow \exp (\bar{\lambda} V)$, and $I^{-1} \circ \exp (-\bar{\lambda} V) \circ I \circ \exp (\bar{\lambda} V)=I$. Since group multiplication and passage to the inverse are continuous operations, this is absurd.

This proves $\lambda_{n} \rightarrow 0$; similarly $\lambda_{n}{ }^{\prime} \rightarrow 0$. Hence it follows that $\exp \left(\lambda_{n}{ }^{\prime \prime} W\right) \rightarrow I$, whence by the preceding paragraph $\lambda_{n}^{\prime \prime} \rightarrow 0$, which completes the proof.

REMARK. It is a corollary of $\$ 5$ alone that $G_{3}{ }^{*}$ is topologically isomorphic in the large with no linear group. (For its generators satisfy the hypotheses of Theorem 2.)

6. The Extended Theorem. Using Theorems 1 and 2, one can exhibit without difficulty a whole class of Lie groups simply isomorphic with no linear group. First we note an almost obvious lemma.

Lemma 2. Any non-Abelian hypercentral Lie group $G$ has infinitesimal generators $U, V, W$ satisfying $[U, V]=W$ and $[X, W]$ $=0$ for every $X$.

Proof. Since $G$ is not Abelian, $U_{0}$ and $V_{0}$ exist satisfying $\left[U_{0}, V_{0}\right]=V_{1} \neq 0$. Moreover either $\left[X, V_{1}\right]=0$ for every $X$, or $\left[U_{1}, V_{1}\right]=V_{2} \neq 0$ for some infinitesimal generator $U_{1}$. But since the Lie algebra of $G$ is nilpotent, the latter alternative cannot recur indefinitely, and so we finally get elements $U_{q}$ and $V_{q}$ such that $\left[U_{q}, V_{q}\right]=V_{q+1}$, while $\left[X, V_{q+1}\right]=0$ for every $X$.

Lemma 3. Any non-Abelian hypercentral Lie group $G$ of matrices has a subgroup $S_{3}$ whose central lies in the central of $G$, topologically isomorphic in the large with $G_{3}$.

Proof. By Lemma 1, the $U, V$, and $W$ of Lemma 2 generate a subgroup $S_{3}$ topologically isomorphic in the large with $G_{3}$, whose central therefore consists of the exp $(\lambda W)$. But since the $\exp (\lambda W)$ commute with all the infinitesimal generators $X$ of $G$, and $G$ (being connected) is generated by these, the $\exp (\lambda W)$ lie in the central of $G$.

THEOREM 3. Any non-Abelian hypercentral Lie group $G$ of matrices is locally topologically isomorphic with a Lie group $G / N$ which is simply isomorphic with no linear group.

Proof. Refer to Lemma 3, and let $N$ be the subgroup of the 
$\exp (n W), n$ any integer. Then $N$ is a discrete subgroup of the central of $G$, and so (see [1], p. 12) $G / N$ is a Lie group locally topologically isomorphic with $G$.

But the homomorphism $G \rightarrow G / N$ carries $S_{3}$ into $S_{3} / N$, which is simply isomorphic with $G_{3} / N=G_{3}{ }^{*}$. This and the corollary to Theorem 1 complete the proof.

E. Cartan [5] has shown that the universal covering group of the group of projective transformations of the line is topologically isomorphic in the large with no linear group.

\section{BIBLIOGRAPHY}

[1] E. Cartan, Théorie des groupes finis et continus et l'analysis situs, Mémorial des Sciences Mathématiques, no. 42, 1930.

[2] L. P. Eisenhart, Continuous Groups of Transformations, 1933.

[3] W. Mayer and T. Y. Thomas, Foundations of the theory of continuous groups, Annals of Mathematics, vol. 36 (1935), pp. 770-822.

[4] A. Speiser, Gruppentheorie, 2d ed., 1927.

[5] La Topologie des Groupes de Lie, Paris, 1936, p. 18.

Society of Fellows, Harvard University

\section{CHARACTERISTICS OF BIRATIONAL TRANSFORMS IN $S_{r}$}

BY B. C. WONG

1. Introduction. Consider a $k$-dimensional variety, $V_{k}^{n}$, of order $n$ in an $r$-space, $S_{r}$. Let us project $V_{k}^{n}$ from a general $(r-k-t-1)$-space of $S_{r}$ upon a general $(k+t)$-space of $S_{r}$ and denote the projection by ${ }_{t} V_{k}^{n}$. We are supposing that $1 \leqq t \leqq k$. Then upon ${ }_{t} V_{k}^{n}$ lies a double variety, $D_{k-t}$, of dimension $k-t$ and order $b_{t}$ and upon $D_{k-t}$ lies a pinch variety, $W_{k-t-1}$, of dimension $k-t-1$ and order $j_{t+1}$. Since the symbol $W_{-1}$ is without meaning, we thus obtain $2 k-1$ characteristics $b_{1}, b_{2}, \cdots, b_{k}$, $j_{2}, j_{3}, \cdots, j_{k}$. The symbol $j_{1}$ has a meaning which will be explained subsequently.

Now let a general $(r-k+q-2)$-space, $S_{r-k+q-2},(1 \leqq q \leqq k)$, be given in $S_{r}$. Through this $S_{r-k+q-2}$ pass $\infty^{k-q+1}$ primes of $S_{r}$ and $\infty^{k-q}$ of these are tangent to $V_{k}^{n}$. The points of contact form a $(k-q)$-dimensional variety, $U_{k-q}$. Denote its order by $m_{q}$. Thus 